\title{
MANAGEMENT OF WHITE SPOT LESIONS USING RESIN INFILTRATION AND MICROABRASION A COMPARATIVE IN VITRO STUDY
}

\author{
Dina M.Abd Elkader ${ }^{1}$, Hanan A. Ismail ${ }^{2}$, \\ Mohamed I. Mowafy ${ }^{3}$, Moustafa N. Abousheleib ${ }^{4}$
}

\section{ABSTRACT}

Study objective: White spot lesions are unwelcomed side effects of fixed appliances that compromise the treatment outcome. Recently the infiltration of white spot lesions has been introduced as a viable treatment alternative and the objective is to evaluate the colour improvement, stability against discolouration and abrasion resistance following resin infiltration and resin infiltration following Microabrasion..

Materials and methods: This study design is an in vitro prospective study that included 45 teeth extracted for orthodontic purposes. Artificial white spot lesions were created in the enamel and teeth were randomly allocated to three groups, then the teeth were treated with infiltration (Icon) and infiltration following Microabrasion (Opalustre) or remained untreated (control). The three groups were then subjected to Thermocycling, tooth 6rush abrasion and discolouration then visible colour change and abrasion resistance were measured.

Results: When $\triangle \mathcal{E}$ was calculated between the baseline measurements and those after white spot lesions there was a clinical and statistical color change in the three groups, in group II (the combined treatment group) the calculated DE between baseline measurements and those taken after treatment and after aging the mean color difference was less than that in group III (only resin infiltration group). Under SEM after aging all the treated surfaces of the specimens in group II were smooth with no microcracks while in group III the treated surfaces showed smooth areas and other areas showed microfissures and microcracks.

Conclusions: When microabrasion preceded resin infiltration in the treatment of WSLs, it improved the color matching immediately after treatment application and showed better performance after aging procedures. It also produced smooth surface free from microcracks under SEM.

Keywords: Microabrasion, Opalustre, Resin infiltration, Icon, CIELAB color analysis, Spectrophotometry, SEM, Thermocycling, Mechanical abrasion.

\section{INTRODUCTION}

Fixed orthodontic treatment offers a lot of benefits to patients such as improved ability to chew food and reduced teeth cavitation and periodontal diseases through achieving proper teeth alignment, alleviation of speech impairment, improved esthetics and providing

\footnotetext{
${ }^{1}$ Researcher, Department of Orthodontics, Faculty of Dentistry, Alexandria University, Alexandria, Egypt.

${ }^{2}$ Professor, Department of Orthodontics, Faculty of Dentistry, Alexandria University, Alexandria, Egypt.

${ }^{3}$ Assistant Professor, Department of Orthodontics, Faculty of Dentistry, Alexandria University, Alexandria, Egypt.

${ }^{4}$ Professor, Department of Biomaterials, Faculty of Dentistry, Alexandria University, Alexandria, Egypt
} 
a confident attractive smile boosting one's self esteem.

Unfortunately, these obtained results may be compromised by enamel demineralization that may occur on the facial surface of anterior teeth due to fixed orthodontic appliances. Orthodontic appliances increase the difficulty of following oral hygiene instructions due to increasing number of plaque retention sites and hindering access to tooth surfaces. Decalcification of the enamel surface adjacent to fixed orthodontic appliances is prevalent as proved be Bishara and Otsby, (2008) mentioning that Decalcification is manifested as a white spot lesion (WSL), if white spot lesions progression was not prevented presenting a problem to the patient then restorative treatment will be required (Bishara, \&Ostby, 2008). Recently, infiltration technology of caries lesions with low-viscosity light curing resins is considered the treatment of choice for early non-cavitated lesions that are not expected to arrest or re-mineralize so that the infiltrants penetrate the porous lesion body of initial caries lesions preventing caries progression.

Laboratory experiments showed significantly deeper penetration of infiltrants into the lesion body than what is seen with conventional adhesives due to their rapid capillary penetration, very low viscosity and high surface tension (Paris, \& MeyerLueckel, 2010). Where in previous studies, it has been proved that resin infiltration had deeper penetration and also it has improved the color appearance of the WSLs as what was proved by the spectrophotometer.

The main objective of the resin infiltration concept is to penetrate the microporosities in the subsurface lesion up to a $450 \mu \mathrm{m}$ depth with a very low viscosity and high penetration coefficient resin (Tirlet, Chabouis, \&Attal,
2013). The porosities that are found in WSLs are responsible for changing the refractive index of the lesion giving it an unacceptable appearance.Whereas the refractive index of enamel is 1.62 units, where as that of white spot lesions with water is 1.33 units or with air it is 1 unit. In this case the loss is only the pseudointact surface enamel, which has to be etched in order to make the hypomineralized pore volume of the lesion body accessible.

So this study was carried-out aiming to compare the effect of resin infiltration technique when combined with microabrasion and when performed alone in management of enamel white spot lesions regarding: Immediate color improvement, color stability of the material, abrasion resistance of the material.

\section{MATERIALS AND METHODS}

This study included freshly extracted teeth that were subjected to decalcification solution in order to induce artificial white spot lesions, then those teeth were randomly assigned to three groups; Group I (control), Group II (microabrasion then resin infiltration) and Group III (resin infiltration alone).

Sample selection: The Sample size was estimated to be 45 premolars that were freshly extracted for orthodontic purposes and were randomly assigned into three groups.

Preparation of the specimens: Teeth were stored in distilled water (Abdullah, ElHarouni, \&Yakout, 2015) for no longer than six months. A mold was fabricated using putty coated with black matte nail polish containing an opening in its center so that when teeth were inserted sequentially in the mold the opening will be facing the middle third of the left half of the crown of each tooth every time to ensure measuring the same point of the crown for the Spectrophotometric measurements reliability in 
every time, upon taking measurements the measured specimen in its mould was put in a dark box to prevent light reflection from affecting the color measurements.

Then the specimens were immersed in a demineralizing solution (Montasser, ElWassefy, \&Taha, 2015) consisting of 2.2 $\mathrm{mmol} / \mathrm{L} \mathrm{Ca} 2+, 2.2 \mathrm{mmol} / \mathrm{L} \mathrm{PO} 43-$ and 50 $\mathrm{mmol} / \mathrm{L}$ acetic acid at $\mathrm{PH} 4.4$ (measured by PH-Meter daily) for 10 days. After immersion and induction of white spot lesions teeth were randomly assigned to three groups and numbered from 1-45 (1-15group I), (16-30 group II) and (31-45 group III).

Group I: Untreated enamel (control).

Group II:Microabrasion combined with resin infiltration.

Group III: Resin infiltration.

Teeth numbered from 1-15 (GP I) were kept untreated in artificial saliva solution (20 $\mathrm{mmol} / \mathrm{L} \mathrm{NaHCO} 3,3 \mathrm{mmol} / \mathrm{L} \mathrm{NaH} 2 \mathrm{PO} 4$ and $1 \mathrm{mmol} / \mathrm{L} \mathrm{CaCl} 2$ at neutral $\mathrm{PH}$ ) (Montasser et al., 2015) throughout the period of the experiment, teeth numbered from 16-30 (GP II) were subjected to two treatment approaches sequentially microabrasion then resin infiltration.

Microabrasion had been performed using Opalustre (Ultradent, Utah, USA), it contains $6.6 \%$ hydrochloric acid slurry and silicon carbide micro-particles providing chemical stain removal along with gentle mechanical abrasion (Yetkiner et al., 2014).

After that resin infiltration was performed using ICON (DMG, Hamburg, Germany) according to manufacturer's instructions.

The ICON kit contains three syringes:

\section{ICON-Etch which contains $15 \%$ hydrochloric acid}

\begin{abstract}
$\checkmark \quad$ ICON-Dry which is composed of Ethanol to improve the efficacy of penetration of the hydrophobic infiltrant (TEGDMA)
\end{abstract}

\section{ICON-Infiltrant which is composed of tetraethylene glycol dimethacrylate, initiator and additives.}

Different procedures were carried-out to simulate the oral environment and applied to GP I, GP II and GP III. First, the three groups were subjected to thermocycling process where the samples were put into thermocycling machine experiencing 10000 cycles which are equivalent to thermal aging for 1 year, teeth were cycled between $5^{\circ} \mathrm{C}$ and $55^{\circ} \mathrm{C}$ in deionized water with a dwell time of 30 seconds and transfer time of 10 seconds (Son, Lim, Ma, \& Park, 2013).

Second, the specimens in the three groups were subjected to mechanical (tooth brush) abrasion where 10000 cycles were applied which corresponds to brushing 3 times daily for a year (Poggio, Beltrami, Scribante, Colombo, \&Chiesa, 2012).

Third, teeth in the three groups were immersed in black tea solution prepared by immersing two prefabricated tea bags $(2.2 \mathrm{~g})$ into $500 \mathrm{ml}$ of boiling distilled water for 10 minutes then the specimens were dipped for 20 minutes once a day (every 24 hour) for 14 days (Poggio, Beltrami, Scribante, Colombo, \&Chiesa, 2012). And, finally the tested specimens were kept outside the artificial saliva solution to dry in order to eliminate any outgasing from organic contamination and water to be put under SEM so that observations of the sample morphology in the coded specimens were performed in a Jeol JSM- 5300 scanning electron microscope operated between 15 and $20 \mathrm{KeV}$.

Color Analysis: The color measurements were taken according to the (Commission 
Internationale de l'Eclairage) CIELAB color space model where:

$\mathrm{L}^{*}$ corresponds to value or degree of lightness

$\mathrm{a}^{*}$ corresponds to red-green color components

b* corresponds to yellow-blue color components

Color Measurements: Color measurements were taken using Vita Easyshade Spectrophotometer, four color measurements were taken for each specimen, at the beginning of the study a base line color measurement was taken for all the specimens and recorded as (T1). Then, Intra-examiner reliability was verified by randomly selecting and examining 10 samples twice before proceeding with the rest of the steps by the same operator after 1 week from the base line readings that were taken before.

Color measurements were taken and recorded as (T2) after induction of white spot lesions on the left half of the crown of each specimen where decalcification occurred. Then, for the third time Spectrophotometric color measurements were taken and recorded as (T3) after the different treatment applications for specimens in group II and group III. Finally, Spectrophotometric measurements were assessed for the last time after different aging procedures were carriedout and were recorded as (T4) using the same tools to check the stability of the color improvement that was achieved. Then the visible color change $(\Delta \mathrm{E})$ was calculated.

Statistical analysis: Normality was checked using descriptive statistics, plots (histogram and box plot) and Shapiro Wilk test. CIE $L^{*} a^{*} b^{*}$ values and $\Delta E$ was presented using mean and standard deviation.
Intra-class correlation coefficient (ICC) was applied to test reliability for mean color change $\left(\Delta \mathrm{E}^{*}\right)$. It was found that intra-examiner ICC was 0.99 with $95 \%$ CI (0.98-0.99).

\section{RESULTS}

After calculating the mean values of $\Delta \mathrm{E}$ between the different time points in the three groups It was found that when $\Delta \mathrm{E}$ was calculated between the baseline measurements and those after white spot lesions there was a clinical and statistical color change in the three groups but the natural teeth didn't respond to the demineralizing solution in the same way where each of the three groups recorded different value of mean color change. Then at (T1-T3) in group I there was no change since there was no treatment applied, in group II (the combined treatments group) and group III (the only resin infiltration group) the mean color difference was recorded as 10.22 in group II (the combined treatments group) which is less than group III (the only resin infiltration group) where the mean color difference was 15.27. And when the baseline measurements were compared to those taken after different aging procedures it was found that the mean color difference was 14.94 in the combined treatments group which is less than the only resin infiltration group where the mean color difference was 23.59 and the untreated enamel group with a mean color difference 23.50.

In group I the major color change after aging was due to the change in $\Delta \mathrm{L}$ while there was no significant change in $\Delta \mathrm{L}, \Delta \mathrm{a}$ or $\Delta \mathrm{b}$ at the other time points. In group II there was color difference after treatment and after aging which was mainly attributed to change in $\Delta \mathrm{L}$ after treatment application and after aging in addition to slight change in $\Delta \mathrm{b}$ after aging procedures. While in group III the color change after treatment was attributed to a change in $\Delta \mathrm{b}$ 
while the color change after aging was attributed to changes in $\Delta \mathrm{L}, \Delta \mathrm{a}$ and $\Delta \mathrm{b}$.

Table 1: Mean values (SD) of $\Delta \mathbf{E}^{*}, \Delta \mathbf{L}^{*}, \Delta \mathbf{a}^{*}$ and $\Delta \mathbf{b}^{*}$ between each of the following pairs of measurement points: baseline (T1), After WSL (T2), after treatment (T3) and after aging (T4)

\begin{tabular}{|c|c|c|c|c|}
\hline & & T1-T2 & T1-T3 & T1-T4 \\
\hline & & \multicolumn{3}{|c|}{ Mean (SD) } \\
\hline \multirow{4}{*}{$\begin{array}{l}\text { Control } \\
(n=15)\end{array}$} & $\Delta \mathrm{L}$ & $-1.09(7.87)$ & $0.03(6.24)$ & $15.77(16.20)$ \\
\hline & $\Delta \mathrm{a}$ & $-0.05(2.30)$ & $-0.21(2.47)$ & $-3.30(4.60)$ \\
\hline & $\Delta \mathrm{b}$ & $0.76(5.93)$ & $0.25(6.70)$ & $-4.66(12.23)$ \\
\hline & $\Delta \mathrm{E}$ & $8.92(4.37)$ & $8.10(4.44)$ & $23.50(11.96)$ \\
\hline \multirow{4}{*}{$\begin{array}{l}\text { Resin infiltration } \\
\text { and microabrasion } \\
(\mathbf{n}=15)\end{array}$} & $\Delta \mathrm{L}$ & $-1.17(4.87)$ & $-9.07(4.10)$ & $11.61(10.31)$ \\
\hline & $\Delta \mathrm{a}$ & $-0.70(0.85)$ & $1.48(1.29)$ & $-2.83(4.19)$ \\
\hline & $\Delta \mathrm{b}$ & $1.06(3.52)$ & $-0.55(4.74)$ & $-5.65(4.44)$ \\
\hline & $\Delta \mathrm{E}$ & $5.49(2.78)$ & $10.22(4.46)$ & $14.94(9.57)$ \\
\hline \multirow{4}{*}{$\begin{array}{c}\text { Resin infiltration } \\
(n=15)\end{array}$} & $\Delta \mathrm{L}$ & $7.47(6.98)$ & $-1.62(4.91)$ & $20.65(13.18)$ \\
\hline & $\Delta \mathrm{a}$ & $-1.73(2.47)$ & $1.29(2.10)$ & $-6.02(4.83)$ \\
\hline & $\Delta \mathrm{b}$ & $0.69(4.66)$ & $12.76(11.40)$ & $-5.77(5.19)$ \\
\hline & $\Delta \mathrm{E}$ & $10.33(5.07)$ & $15.27(9.37)$ & $23.59(12.61)$ \\
\hline
\end{tabular}

\section{Surface Morphology under SEM}

After Spectrophotometric color measurements randomly nine selected specimens from the three groups (three specimens from each group) were prepared to be scanned under SEM in order to qualitatively assess the surface morphology of the specimens surfaces after the aging challenges.

In group I the untreated enamel surface showed increased microcracks and microfissures all over the tested area. In group II the treated enamel surface using microabrasion and resin infiltration the resin was still present on the surface and showed the least microcracks and microfissures where the surface of all the treated area appeared to be smooth. In group III the treated enamel area using only resin infiltration material the resin was also still present on the surface and showed areas with microcracks and microfissures along with smooth areas showing minimal microfissures differentially indicating better resistance to abrasion and aging challenges in group II. 


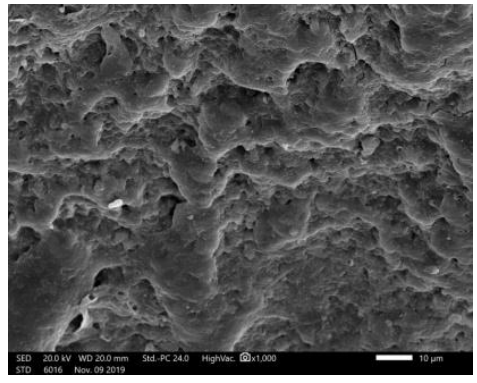

Group I

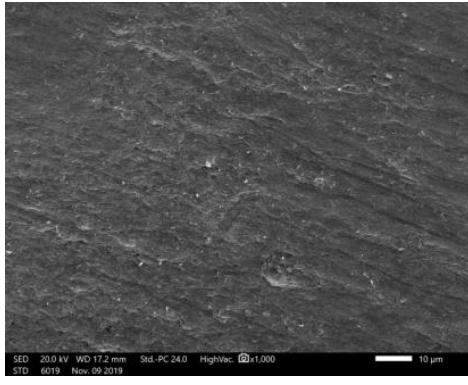

Group II

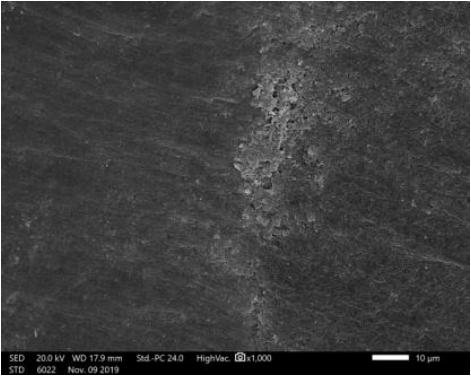

Group III

Figure 1: The surface morphology under SEM in the three groups (At 1000X)

\section{DISCUSSION}

This study investigated the immediate color improvement of the white spot lesions that were induced in sound human enamel after treatment with either microabrasion followed by resin infiltration or resin infiltration alone, it also investigated the long term color stability of both treatment approaches following different aging factors such as thermocycling, mechanical abrasion and extrinsic staining and finally it investigated the difference in abrasion resistance of both treatments.

In this study there were three study groups; group (control), group II (microabrasion then resin infiltration treatment were applied to the artificially induced WSLs) and group III (only resin infiltration treatment was applied). Color changes were recorded using Vita Easyshade spectrophotometer according to CIE L*a*b* color analysis at four time points; $\mathrm{T} 1$ at baseline, $\mathrm{T} 2$ after induction of WSLs, T3 after treatment application and T4 after aging procedures. Results revealed that there was a difference between the initial color teeth and the color recorded after decalcification and induction of WSLs in the three groups and it was found that the color change occurred didn't happen in the same manner or with similar values where in group I and II $\mathrm{L}^{*}$ value slightly decreased rendering the tested area darker while in group III L* increased so lightness increased indicating different responses from the natural teeth which might be explained due to the difference in the initial teeth color and their different mineral content, in group II (the combined treatment group) the color difference that was recorded after treatment compared to the baseline measurements was mainly attributed to the decrease in $\mathrm{L}^{*}$ value after treatment so tested area became darker and its increase after aging procedures making the surface lighter again and more blue due to the decrease in $b^{*}$ value showing less color change in group II than that in the only resin infiltration group indicating a better color matching and improvement after application of the microabrasive material (Opalustre) followed by resin infiltration using ICON than using resin infiltration alone where teeth turned more blue after resin infiltration treatment due to the decrease in $b^{*}$ value. And after the aging procedures were implemented simulating the oral cavity conditions equivalent to 1 year, the results showed better performance and less deterioration after aging procedures in group II with the combined treatments than with the resin infiltration alone since the mean color difference recorded between baseline measurements and measurements taken after aging in the combined treatments group was less than that in the resin infiltration group where there was a change in the $\mathrm{L}^{*} \mathrm{a} * \mathrm{~b}^{*}$ color 
compononents. Also in the untreated enamel group there was severe deterioration after aging close to that in the resin infiltration group.

The results of the current study were similar to other studies that were carried out stating that resin infiltrated surfaces showed significantly higher color alteration than sound enamel as concluded by Zhao and Ren (2016) who attributed this color change to the decrease in $\Delta \mathrm{L}$ decreasing lightness, when measurements were taken by spectrophotometer in this study the color change $(\Delta \mathrm{E})$ in the resin infiltration group was attributed to changes in $\Delta \mathrm{L}, \Delta \mathrm{a}$ and $\Delta \mathrm{b}$ and this may be because of one of two reasons. First, the color measurements in this study were taken after thermocycling, mechanical abrasion and extrinsic staining while in the other study color measurements were taken after surface staining only. Second, each specimen presents its own color change pattern according to its original color and mineral content.

Also, when specimens in the previous study was put under SEM resin infiltrated surfaces appeared intact and uniform before aging challenges while surface microcracks and microfissures appeared following thermocycling and water storage procedures which may render the surface susceptible to discoloration which is also similar to the findings in the our study in the resin infiltration group.

Our results were also consistent with the results of a study by Leland, Akyalcin, English, Tufekci and Paravina, (2016) who stated that enamel treated with resin infiltration was more susceptible to discoloration than regular enamel when exposed to various staining agents relating this extrinsic staining susceptibility to the type of resin matrix used.
Some other studies stated different results after comparing resin infiltration and microabrasion treatment approaches such as what was said by Gu et al., (2019) showing thatthere was no significant color change in the resin infiltration group over 12 months while in the microabrasion group there was a significant decrease in $\Delta \mathrm{E}$ indicating that resin infiltration had an immediate and long term esthetic improvement of WSLs that was stable, where asmicroabrasion showed significant improvement during the follow up period and these results may be attributed to that the previous study was in-vivo clinical experiment where the resin infiltrant was allowed to be applied after three months from debonding of the fixed orthodontic appliance giving a chance for remineralization of the WSLs by the salivary ions improving their appearance, also it was reported that some of the lesions disappeared within several weeks.

In another in-vitro study by Yetkiner, Wegehaupt, Wiegand, Attin, \&Attin, 2014) to evaluate the colour improvement of WSLs and their stability against discolouration following infiltration, fluoride, or microabrasion treatments where it revealed that only infiltration reduced this effect to baseline as the highest $\Delta \mathrm{E}$ improvement was obtained by infiltration then micro-abrasion followed by fluoride. This improvement was stable only for infiltration during discoloration and that may contradict the results of our study because only discoloration challenge was applied to the specimens with no thermal or mechanical aging which are necessary in simulating the oral cavity conditions compromising the results of the previous study.

In our study when resin infiltration material was applied to specimens and it was compared to specimens with microabrasion followed by resin infiltration as what was performed, the 
results showed that the combined microabrasion-resin infiltration treatments provided better color matching and when tested after aging procedures the specimens showed better performance in oral cavity simulation conditions and better long term stability after thermocycling, mechanical abrasion and discoloration.

\section{CONCLUSIONS}

Based on the previous results, it was concluded that:

1- Natural teeth don't respond to demineralizing solution in the same way.

2- The untreated enamel showed no significant color change at first, but it showed severe color change and deterioration after aging

3- When microabrasion preceded resin infiltration in the treatment of WSLs, it improved the color matching immediately after treatment application and increased the color stability and showed better performance of the material against aging challenges.

4- The combined treatment approach rendered the treated enamel surface smooth and free from microfissures and microcracks indicating better wear and abrasion resistance of the material.

\section{REFERENCES}

Abdullah, E. M., El-Harouni, N. M., \&Yakout, Y. M. (2015).Effect of Nanohydroxyappetite versus Casein phosphopeptide amorphous calcium phosphate on remineralization of early enamel lesions- Invitrostudy.Egyptian Orthodontics Journal, 48, 71-84.
Bishara, S. E., \&Ostby, A. W. (2008). White spot lesions: formation, prevention, and treatment. In Seminars in Orthodontics, 14(3), 174-182.

Gu, X., Yang, L., Yang, D., Gao, Y., Duan, X., Zhu, X., ...\& Li, J. (2019). Esthetic improvements of postorthodontic white-spot lesions treated with resin infiltration and microabrasion: A splitmouth, randomized clinical trial. The Angle Orthodontist, 89(3), 372-377.

Leland, A., Akyalcin, S., English, J. D., Tufekci, E., \&Paravina, R. (2016). Evaluation of staining and color changes of a resin infiltration system. The Angle Orthodontist, 86(6), 900-904.

Montasser, M. A., El-Wassefy, N. A., \&Taha, M. (2015).In vitro study of the potential protection of sound enamel against demineralization.

Progress in orthodontics, 16(1), 12.

Paris, S., \& Meyer-Lueckel, H. (2010).Infiltrants inhibit progression of natural caries lesions in vitro. Journal of dental research, 89(11), 1276-1280.

Poggio, C., Beltrami, R., Scribante, A., Colombo, M., \&Chiesa, M. (2012). Surface discoloration of composite resins: Effects of staining and bleaching. Dental research journal, 9(5), 567.

Son, J. H., Lim, D. S., Ma, D. S., \& Park, D. Y. (2013). Abrasion of resin infiltrated enamel by tooth brushing. Journal of Korean Academy of Oral Health, 37(1), 9-15.

Tirlet, G., Chabouis, H. F., \&Attal, J. P. (2013). Infiltration, a new therapy for masking enamel white spots: a 19-month follow-up case series. European Journal of Esthetic Dentistry, 8(2), 180-190. 
Yetkiner, E., Wegehaupt, F., Wiegand, A., Attin, R., \&Attin, T. (2014). Colour improvement and stability of white spot lesions following infiltration, microabrasion, or fluoride treatments in vitro. European journal

of orthodontics, 36(5), 595-602.

Zhao, X., \&Ren, Y. F. (2016). Surface properties and color stability of resin-infiltrated enamel lesions. Operative dentistry, 41(6), 617-626. 